Discrete Comput Geom 26:375-386 (2001)

DOI: $10.1007 / \mathrm{s} 00454-001-0036-9$

\title{
On the Pentomino Exclusion Problem
}

\author{
S. Gravier and C. Payan
}

CNRS, Laboratoire Leibniz,

46 avenue Félix Viallet, 38031 Grenoble Cedex 1, France

\begin{abstract}
In this paper we are interested in the Pentomino Exclusion Problem due to Golomb: Find the minimum number of unit squares to be placed on a $k \times n$ chessboard so as to exclude all pentominoes. Using an appropriate definition of density of a tiling, we obtain the asymptotic value of this number, and we establish this number for the $k \times n$ chessboard when $k \leq 4$.
\end{abstract}

\section{Introduction}

A polyomino is a pattern formed by the connection of a specified number of equal-sized squares along common edges (see [2]). A pentomino is a polyomino composed of five squares. The interior boundary $\delta_{\text {int }}(P)$ of a polyomino $P$ is the set of squares of $P$ having a common edge with the "exterior" of $P$. The exterior boundary $\delta_{\text {ext }}(P)$ of a polyomino $P$ is the interior boundary of the complement of $P$. The perimeter of a polyomino $P$ is $\left|\delta_{\text {int }}(P)\right|$. For a given polyomino $P, \Delta(P)$ denotes the area of $P$.

Consider the adjacency relations $\alpha$ and $\beta$, which defines what is usually called respectively 8-connectivity and 4-connectivity in discrete geometry, between squares in $\mathbb{Z}^{2}$ : write $C \alpha C^{\prime}$ (resp. $C \beta C^{\prime}$ ) iff $C$ and $C^{\prime}$ have a common vertex (resp. edge).

For a given polyomino $P$, we can build a graph $G(P)=(V, E)$ defined by $V=$ $\{p \mid p$ is the center of a unit square in $P\}$ and $E=\{U V \mid U \alpha V\}$. A vertex $v$ of $G(P)$ can be seen as a square of $\mathbb{R}^{2}$, so for brevity sometimes $v$ should be seen as the unique corresponding square (see Fig. 1). Moreover, $G\left(\mathbb{Z}^{2}\right)$ is in graph-theoretical language usually called the total infinite complete grid graph which can be defined as a total product of two infinite paths.

Golomb [2] proposed the following Pentomino Exclusion Problem, denoted $\left(P E P_{k \times n}\right)$ : Find the minimum number of unit squares to be placed on a $k \times n$ chessboard so as to exclude all pentominoes.

In a previous paper [3] we introduced a notion of density of a tiling with (for example) 


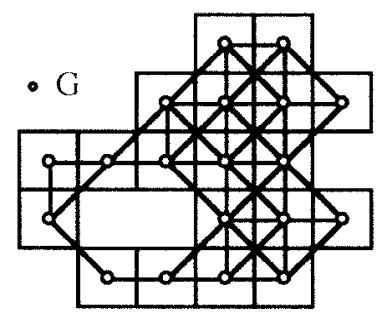

Fig. 1. A polyomino $P$, the graph $G(P)$.

polyominoes. Using this definition in the next section, we determine the asymptotic value (i.e., the density of a tiling where the tiles are polyominoes composed of less than five squares) of the Pentomino Exclusion Problem and other related problems.

Finally, in Section 3, we investigate $\left(\mathrm{PEP}_{k \times n}\right)$ problems when $k=4$.

\section{Assymptotic Results}

In order to state our results we need some preliminary definitions.

We denote by $\left(\mathrm{G}_{\Delta}\right)$ the problem:

Find the minimum density of unit squares to be placed on the plane so as to exclude all polyominoes of area $>\Delta$.

For instance, the Pentomino Exclusion Problem in the plane is equivalent to the problem $\left(\mathrm{G}_{4}\right)$.

An admissible solution of $\left(\mathrm{G}_{\Delta}\right)$ is a set $\mathcal{S}$ of squares centered on $\mathbb{Z}^{2}$ such that any connected component in the $\beta$ adjacency of $\mathbb{R}^{2}-\mathcal{S}$ has area less than or equal to $\Delta$. The squares belonging to an admissible solution $\mathcal{S}$ are filled in (i.e. black) and the others are left white.

We now need a measure, called "density," defined on an admissible solution of $\left(\mathrm{G}_{\Delta}\right)$ in order to compare two admissible solutions. If $T$ is a finite subset of $\mathbb{Z}^{2}$, a natural way to define the density of $\mathcal{S}$ relative to $T$ is $|\mathcal{S} \cap T| /|T-\mathcal{S}|$. We now show a way to extend this definition to the infinite case:

For an admissible solution $\mathcal{S}$ of $\left(\mathrm{G}_{\Delta}\right)$, observe that if we remove one "crossing edge" of each $K_{4}$ (complete graph on four vertices) in $G(\mathcal{S}$ ), then the resulting plane graph $G^{\prime}(\mathcal{S})$ defines a tiling of $\mathbb{R}^{2}$ (see Fig. 2) where the tiles are the faces of $G^{\prime}(\mathcal{S})$. For a face (or a tile) $\langle C\rangle$ of $G^{\prime}(\mathcal{S})$ there corresponds a unique polyomino $C$ where $\delta_{\text {ext }}(C) \subset \mathcal{S}$. Some of these tiles correspond to some connected components (in the $\beta$ connectivity) of $\mathbb{Z}^{2}-\mathcal{S}$. Other tiles are triangles corresponding to three mutually adjacent elements of $\mathcal{S}$ (in this case $C=\emptyset$ ).

Let $D$ be a finite subset of $\mathbb{R}^{2}$. The density of an admissible solution $\mathcal{S}$ of $\left(\mathrm{G}_{\Delta}\right)$ relative to $D$ is

$$
d(\mathcal{S}, D)=\frac{\text { black area of } \bar{D}}{\text { white area of } \bar{D}}
$$




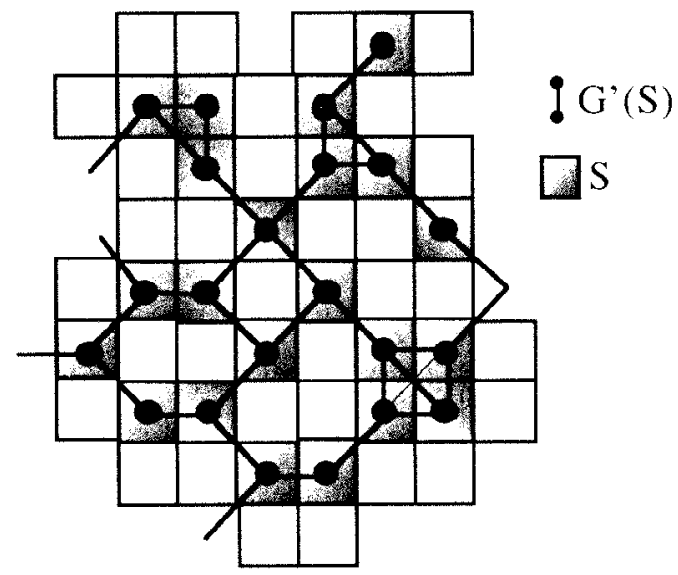

Fig. 2. $\mathcal{S}$ and $G^{\prime}(\mathcal{S})$.

where $\bar{D}$ is the union of all faces of $G^{\prime}(\mathcal{S})$ which intersect $D$. Notice that $\bar{D}$ defines a polyomino $P$ with unit squares from $\mathcal{S}$, and each square in the interior boundary of $P$ belongs to $\mathcal{S}$. Moreover, observe that $d(\mathcal{S}, D)$ is well-defined since each face of $G^{\prime}(\mathcal{S})$ define a polyomino with bounded area.

Let $B_{r}$ be a ball of $\mathbb{R}^{2}$ of radius $r$. Then

$$
\underline{d}(\mathcal{S})=\liminf _{r \rightarrow \infty} d\left(\mathcal{S}, B_{r}\right) \quad \text { and } \quad \bar{d}(\mathcal{S})=\limsup _{r \rightarrow \infty} d\left(\mathcal{S}, B_{r}\right),
$$

are called the lower and upper density, respectively. If these two values coincide, their common value is called density $d(\mathcal{S}, D)$. This kind of definition of density is more or less standard (see, for example, [4]). In [3] we proved:

Theorem 1. Let $\Delta_{n}$ be the maximum number of squares belonging to a polyomino of perimeter $n$ with $n=4 q+r>0$ and $0 \leq r \leq 3 ; \Delta_{n}$ is given by the following function:

$$
\Delta_{n}=\left\{\begin{array}{lll}
2 q^{2}+2 q+1 & \text { if } \quad r=0, \\
2 q^{2}+3 q+1 & \text { if } \quad r=1, \\
2 q^{2}+4 q+2 & \text { if } \quad r=2, \\
2 q^{2}+5 q+3 & \text { if } \quad r=3
\end{array}\right.
$$

Theorem 2. Let $n=4 q+r \geq 5$, with $0 \leq r \leq 3$, be an integer such that $\Delta \geq \Delta_{n}$. If $q>1$ and $\Delta-\Delta_{n} \leq\lceil q / 2\rceil$, then an optimal solution $\mathcal{S}$ of $\left(G_{\Delta}\right)$ satisfies

$$
\underline{d}(\mathcal{S}) \geq \frac{(n+4) / 2-1}{\Delta_{n}} .
$$

For any $\Delta \geq \Delta_{n}$, we have

$$
\bar{d}(\mathcal{S}) \leq\left\{\begin{array}{lll}
\frac{(n+4) / 2-1}{\Delta_{n}} & \text { if } & r \in\{0,2\}, \\
\frac{(n+5) / 2-1}{\Delta_{n}} & \text { if } & r \in\{1,3\} .
\end{array}\right.
$$


As noticed in [3], a direct consequence of Theorem 2 is that when $r \in\{0,2\}, q>1$ and when $\Delta-\Delta_{n} \leq\lceil q / 2\rceil$ the density of an optimal solution of $\left(\mathrm{G}_{\Delta}\right)$ exists and is equal to

$$
\frac{(n+4) / 2-1}{\Delta_{n}}
$$

Moreover, this density is independent from the position of the ball $B_{r}$.

In this section we complete Theorem 2 for $\Delta \leq 7$ since the first values given by Theorem 2 deal with $\Delta \geq 8$.

Theorem 3. The only admissible solution of $\left(\mathrm{G}_{0}\right)$ is $\mathcal{S}=\mathbb{Z}^{2}$. An optimal solution of $\left(\mathrm{G}_{\Delta}\right)$ satisfies

$$
\begin{aligned}
& d(\mathcal{S})=1 \quad \text { when } \quad \Delta \leq 2 \text {, } \\
& \left.\begin{array}{l}
\bar{d}(\mathcal{S}) \leq 1 \\
d(\mathcal{S}) \geq \frac{5}{6}
\end{array}\right\} \quad \text { when } \quad \Delta=3 \text {, } \\
& d(\mathcal{S})=\frac{3}{4} \quad \text { when } \quad \Delta=4 \text {, } \\
& d(\mathcal{S})=\frac{3}{5} \quad \text { when } \quad \Delta=5 \text {, } \\
& \left.\begin{array}{l}
\bar{d}(\mathcal{S}) \leq \frac{3}{5} \\
d(\mathcal{S}) \geq \frac{7}{12}
\end{array}\right\} \quad \text { when } \quad \Delta=6, \\
& d(\mathcal{S})=\frac{4}{7} \quad \text { when } \quad \Delta=7 \text {. }
\end{aligned}
$$

Proof. Let $\mathcal{S}$ be an optimal solution of $\left(\mathrm{G}_{\Delta}\right)$. Let $D \subset \mathbb{R}^{2}$ and let

$$
\bar{D}=\bigcup_{\left\{\langle C\rangle \in G^{\prime}(\mathcal{S}) \mid\langle C\rangle \cap D \neq \emptyset\right\}}\langle C\rangle .
$$

First we claim that:

$$
\text { It may be assumed that every }\langle C\rangle \text { has no hole. }
$$

If $\langle C\rangle$ has a hole, then move it closer to the exterior boundary of $\langle C\rangle$ in order to obtain a new face $\left\langle C^{\prime}\right\rangle$ with no hole. If we repeat this operation for any $\langle C\rangle$ having a hole, then we obtain a new admissible solution of $\left(\mathrm{G}_{\Delta}\right)$ with the same density.

Now we assume that any face of $G^{\prime}(\mathcal{S})$ has no hole. Using the structure of $\mathbb{Z}^{2}$, we claim that

$$
d(\mathcal{S}, D)=\frac{\sum_{\langle C\rangle \in \bar{D}}\left(\left|\delta_{\mathrm{ext}}(C)\right| / 2-1\right)}{\sum_{\langle C\rangle \in \bar{D}}|C|} .
$$

If $P$ is the polyomino defined by $\bar{D}$, then, by Pick's theorem, we obtain that the area of $\bar{D}$ is given by $\left|P-\delta_{\text {int }}(P)\right|+\left|\delta_{\text {int }}(P)\right| / 2-1$ and the area of each $\langle C\rangle$ is given by $|C|+\left|\delta_{\text {ext }}(C)\right| / 2-1$, since by assumption no $\langle C\rangle$ has a hole. Now by additivity of the area and since $\{\langle C\rangle \mid\langle C\rangle \in \bar{D}\}$ is a tiling of $\bar{D}$, we have that $\sum_{\langle C\rangle \in \bar{D}}\left(\left|\delta_{\text {ext }}(C)\right| / 2-1\right)$ is equal to the number of squares in $\mathcal{S} \cap P$ not in the interior boundary of $P$, plus half the number of squares in the interior boundary of $P$ which corresponds to the black area of $\bar{D}$. Also, $\sum_{\langle C\rangle \in \bar{D}}|C|$ is the number of squares in $P$ not in $\mathcal{S}$, which corresponds to the white area of $\bar{D}$. 


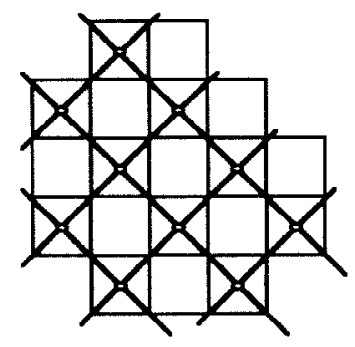

$\Delta=1$

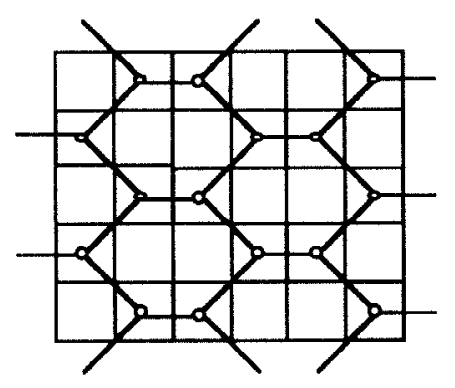

$\Delta \leq 2$

Fig. 3. $1 \leq \Delta \leq 2$.

From (2), we have

$$
d(\mathcal{S}, D) \geq \min _{\langle C\rangle \in \bar{D}} \frac{\left|\delta_{\text {ext }}(C)\right| / 2-1}{|C|} .
$$

Hence, to obtain the lower bounds on $\underline{d}(\mathcal{S})$, it is sufficient to check that

$$
\left|\delta_{\text {ext }}(C)\right| \geq\left\{\begin{array}{lll}
4 & \text { if } & |C|=1, \\
6 & \text { if } & |C|=2, \\
7 & \text { if } & |C|=3, \\
8 & \text { if } & |C|=4, \\
8 & \text { if } & |C|=5, \\
9 & \text { if } & |C|=6, \\
10 & \text { if } & |C|=7 .
\end{array}\right.
$$

To prove the upper bounds on $\bar{d}(\mathcal{S})$ it is sufficient to exhibit tilings having the appropriate density (see Figs. 3-6). To determine the density of the tilings described in Figs. 3-6, it is sufficient to observe that, by (2), we have

$$
d(\mathcal{S}, D) \leq \max _{\langle C\rangle \in \bar{D}} \frac{\left|\delta_{\text {ext }}(C)\right| / 2-1}{|C|}
$$

\section{Finite Cases}

In this section we investigate the problem $\left(\mathrm{PEP}_{k \times n}\right)$ for some values of $k$ and $n$. We denote by $G_{k, n}$ an instance of $\left(\mathrm{PEP}_{k \times n}\right)$. For given $k$ and $n, C_{1}, \ldots, C_{n}$ (resp. $\left.R_{1}, \ldots, R_{k}\right)$ denote the columns (resp. the rows) of $G_{k, n}$. The squares of $G_{k, n}$ are denoted by $s_{i, j}$ where $\left\{s_{i, j}\right\}=R_{i} \cap C_{j}$. A free polyomino of a solution $\mathcal{S}$ of $\left(\mathrm{PEP}_{k \times n}\right)$ is a polyomino which does not intersect $\mathcal{S}$.

First we give some upper bounds on the cardinality of a solution of $\left(\mathrm{PEP}_{k \times n}\right)$ for small values of $k$ and $n$. It is easy to see that:

Lemma 1. Every solution $\mathcal{S}$ to $\left(\mathrm{PEP}_{2 \times 3}\right)$ satisfies $|\mathcal{S}| \geq 2$. 

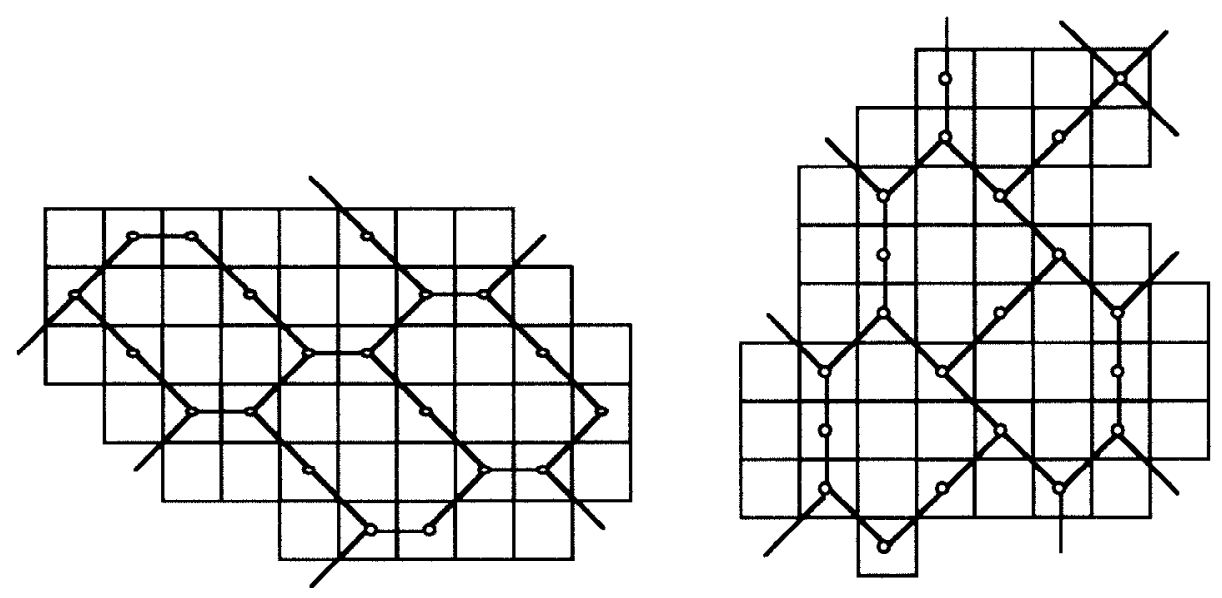

$\Delta=4$

Fig. 4. $\Delta=4$.

Theorem 4. Let $\mathcal{S}_{k, n}$ be an optimal solution of $\left(\mathrm{PEP}_{k \times n}\right)$. Then

$$
\left|\mathcal{S}_{k, n}\right|= \begin{cases}\lfloor n / 5\rfloor & \text { if } \quad k=1 \\ 2\lfloor n / 3\rfloor & \text { if } \quad k=2 \\ n & \text { if } \quad k=3 \text { and } n \geq 2\end{cases}
$$

Proof. When $k=1$, the theorem is obvious. When $k=2$, then Theorem 4 is a direct consequence of Lemma 1 . If $k=3$, then let $\mathcal{S}$ be a solution of $\left(\mathrm{PEP}_{3 \times n}\right)$. We prove the lower bound by induction on $n$. It is easy to see that if $n \leq 3$, then Theorem 4 holds. Assume that $n>3$. By Lemma 1, we have $\left|\mathcal{S} \cap\left(C_{1} \cup C_{2}\right)\right| \geq 2$. Now, Theorem 4 holds by induction hypothesis on $C_{3} \cup \cdots \cup C_{n}$.

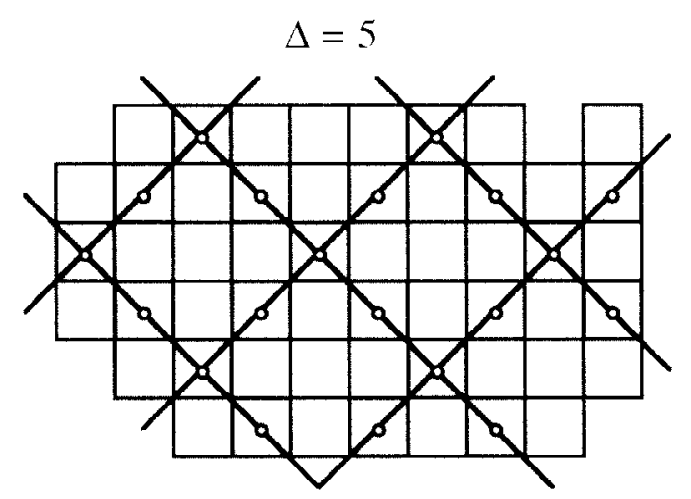

Fig. 5. $5 \leq \Delta \leq 6$. 


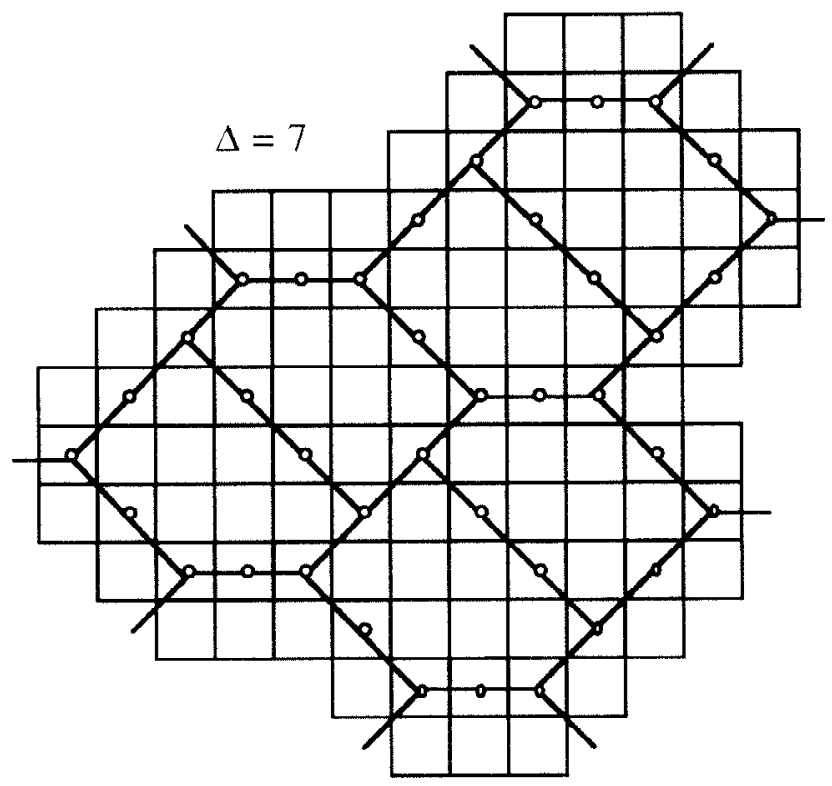

Fig. 6. $\Delta=7$.

To achieve the proof of Theorem 4, we exhibit a solution:

$$
\mathcal{S}=\left\{\begin{array}{cc}
i=2 \text { if } j \text { is even } \\
s_{i, j} \mid \begin{array}{c}
i+j \equiv 0[4], \text { otherwise }
\end{array}
\end{array}\right\}
$$

satisfying $|\mathcal{S}|=n$.

Lemma 2. Every solution $\mathcal{S}$ to $\left(\mathrm{PEP}_{4 \times 4}\right)$ satisfies $|\mathcal{S}| \geq 5$. Moreover, there exists a unique, up to rotation, solution $F$ (see Fig. 7) with only five squares in $\left(\mathrm{PEP}_{4 \times 4}\right)$.

Proof. Let $\mathcal{S}$ be a solution of $\left(\mathrm{PEP}_{4 \times 4}\right)$ with less than six squares. We claim that

$$
\mathcal{S} \cap C_{i} \neq \emptyset \quad \text { and } \quad \mathcal{S} \cap R_{i} \neq \emptyset \quad \text { for all } \quad i=1, \ldots, 4 .
$$

Indeed, assume, in the opposite case, that $\mathcal{S} \cap R_{1}=\emptyset$ or $\mathcal{S} \cap R_{2}=\emptyset$. If $\mathcal{S} \cap R_{2}=\emptyset$,
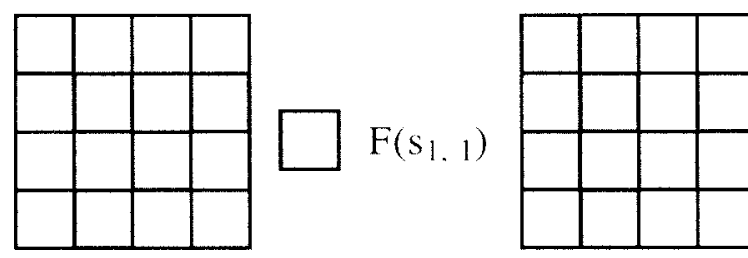

Fig. 7. The solution $F$ of $\left(\mathrm{PEP}_{4 \times 4}\right)$ and a solution $S$. 
then $R_{2} \cup R_{3} \subseteq \mathcal{S}$. If $\mathcal{S} \cap R_{1}=\emptyset$, then $R_{1} \subseteq \mathcal{S}$. Now, by (1), $\left|\mathcal{S} \cap\left(R_{3} \cup R_{4}\right)\right| \geq 2$. In any case, by symmetry, we obtain that $|\mathcal{S}|>5$, a contradiction.

Case 1: none of the squares $s_{1,1}, s_{1,4}, s_{4,1}$ and $s_{4,4}$ belongs to $\mathcal{S}$. In this case, by (5), we may assume that $s_{2,1} \in \mathcal{S}$.

By Lemma 1 applied on $\left(C_{1} \cup C_{2}\right) \cap\left(R_{2} \cup R_{3} \cup R_{4}\right)$ and on $\left(C_{3} \cup C_{4}\right) \cap\left(R_{2} \cup R_{3} \cup R_{4}\right)$, we have $\left|\mathcal{S} \cap\left(R_{2} \cup R_{3} \cup R_{4}\right)\right| \geq 4$. Hence, since $|\mathcal{S}| \leq 5$, we have $s_{1,3} \notin \mathcal{S}$.

By Lemma 1 applied on $\left(C_{1} \cup C_{2}\right) \cap\left(R_{2} \cup R_{3} \cup R_{4}\right)$ and on $\left(C_{3} \cup C_{4}\right) \cap\left(R_{1} \cup R_{2} \cup R_{3}\right)$, we have $\left|\mathcal{S}-\left\{s_{1,1}, s_{1,2}, s_{3,4}, s_{4,4}\right\}\right| \geq 4$. Hence, since $|\mathcal{S}| \leq 5$, we have $s_{4,3} \notin \mathcal{S}$.

By (5), we have $s_{4,2} \in \mathcal{S}$. So, by symmetry and by (5), we may assume that $s_{2,1} \in \mathcal{S}$.

Since $|\mathcal{S}| \leq 5$ and since, by Lemma $1,\left|\left(\mathcal{S} \cap\left(R_{1} \cup R_{2} \cup R_{3}\right)\right) \cap\left(C_{3} \cup C_{4}\right)\right| \geq 2$, we have $\mathcal{S} \cap\left(R_{1} \cup R_{2}\right)=\left\{s_{1,2}, s_{2,1}, s_{4,2}\right\}$. However, now observe that $\left\{s_{3,1}, s_{4,1}, s_{2,2}, s_{3,2}, s\right\}$ induces a free pentomino for any $s \in\left\{s_{2,3}, s_{3,3}\right\}$. Thus $s_{2,3}, s_{3,3} \in \mathcal{S}$.

Since $|\mathcal{S}| \leq 5$, we have that $\left(C_{3} \cup C_{4}\right)-\mathcal{S}$ contains a free pentomino, which contradicts the fact that $\mathcal{S}$ is a solution of $\left(\mathrm{PEP}_{4 \times 4}\right)$.

Case 2: $s_{1,1}$ belongs to $\mathcal{S}$. Assume that $s_{1,1} \in \mathcal{S}$.

By Lemma 1, we have $\left|\left(\mathcal{S} \cap\left(R_{1} \cup R_{2}\right)\right) \cap\left(C_{2} \cup C_{3} \cup C_{4}\right)\right| \geq 2$ and $\mid\left(\mathcal{S} \cap\left(R_{3} \cup\right.\right.$ $\left.\left.R_{4}\right)\right) \cap\left(C_{2} \cup C_{3} \cup C_{4}\right) \mid \geq 2$. Hence, since $|\mathcal{S}| \leq 5$, we obtain that $s_{1,2}, s_{1,3}$ and $s_{1,4}$ do not belong to $\mathcal{S}$. Moreover, by symmetry, we obtain that $s_{2,1}, s_{3,1}$ and $s_{4,1}$ do not belong to $\mathcal{S}$.

By Lemma 1, we have $\left|\left(\mathcal{S} \cap\left(R_{1} \cup R_{2}\right)\right) \cap\left(C_{2} \cup C_{3} \cup C_{4}\right)\right| \geq 2$ and $\mid\left(\mathcal{S} \cap\left(R_{3} \cup\right.\right.$ $\left.\left.R_{4}\right)\right) \cap\left(C_{1} \cup C_{2} \cup C_{3}\right) \mid \geq 2$. Hence, since $|\mathcal{S}| \leq 5$, we obtain that $s_{4,3}$ and $s_{4,4}$ do not belong to $\mathcal{S}$. Moreover, by symmetry, we obtain that $s_{3,4} \notin \mathcal{S}$.

We must have $\left\{s_{2,2}, s_{2,4}, s_{4,2}\right\} \subset \mathcal{S}$. Finally, since $|\mathcal{S}| \leq 5$ and by (5) we must have $s_{3,3} \in \mathcal{S}$, which completes the proof of lemma.

The unique solution, up to rotation, is $\mathcal{S}=\left\{s_{1,1}, s_{2,2}, s_{2,4}, s_{3,3}, s_{4,2}\right\}$.

We denote by $F(s)$ a solution $\mathcal{S}$ of $\left(\mathrm{PEP}_{4 \times 4}\right)$ of cardinality five where $s \in \mathcal{S}$ is a "corner" (see Fig. 7).

In order to study the structure of a solution of $\left(\mathrm{PEP}_{k \times n}\right)$, we now need some additional definitions. A $P(3,4)$ configuration of a solution $\mathcal{S}$ of a $\left(\mathrm{PEP}_{4 \times n}\right)$ (for some $n$ ) is a column $C_{i}$ such that $s_{2, i} \in \mathcal{S}$ and $s_{1, i}$ belong to a white polyomino in $G_{4, i}$ of size 4 and $s_{3, i}, s_{4, i}$ belong to a white polyomino in $G_{4, i}$ of size 3. A $P(4)$ configuration of a solution $\mathcal{S}$ of a $\left(\mathrm{PEP}_{4 \times n}\right)$ (for some $n$ ) is a column $C_{i}$ such that three squares of $C_{i}$ belong to a white polyomino in $G_{4, i}$ of size 4 . Note that the fourth column of an $F(s)$ is either a $P(3,4)$ or a $P(4)$ configuration.

Lemma 3. Let $i \leq n-5$. If $C_{i}$ is a $P(4)$ configuration of $\mathcal{S}$, then $\mid \mathcal{S} \cap\left(C_{i+1} \cup C_{i+2} \cup\right.$ $\left.C_{i+3} \cup C_{i+4}\right) \mid \geq 7$. If $C_{i}$ is a $P(3,4)$ configuration of $\mathcal{S}$, then $\mid \mathcal{S} \cap\left(C_{i+1} \cup C_{i+2} \cup C_{i+3} \cup\right.$ $\left.C_{i+4}\right) \mid \geq 6$. Moreover, if we have $\left|\mathcal{S} \cap\left(C_{i+1} \cup C_{i+2} \cup C_{i+3} \cup C_{i+4}\right)\right|=6$, then $C_{i+4}$ is a $P(3,4)$ or a $P(4)$ configuration (see Fig. 7 ).

Proof. First, suppose that $C_{i}$ is a $P(4)$ configuration. Then at least three squares must belong to $\mathcal{S} \cap C_{i+1}$. Moreover, by Lemma $1,\left|\mathcal{S} \cap\left(C_{i+2} \cup C_{i+3} \cup C_{i+4} \cap\left(R_{1} \cup R_{2}\right)\right)\right| \geq 2$ and $\left|\mathcal{S} \cap\left(C_{i+2} \cup C_{i+3} \cup C_{i+4} \cap\left(R_{3} \cup R_{4}\right)\right)\right| \geq 2$, so we obtain that $\mid S \cap\left(C_{i+1} \cup C_{i+2} \cup\right.$ $\left.C_{i+3} \cup C_{i+4}\right) \mid \geq 7$. 
Now suppose that $C_{i}$ is a $P(3,4)$ configuration. Then $s_{1, i+1} \in \mathcal{S}$. We claim that:

$$
\text { We may assume that }\left|\mathcal{S} \cap C_{i+1}\right| \leq 2 \text {. }
$$

Indeed, in the opposite case by Lemma 1 applied on $C_{i+2} \cup C_{i+3} \cup C_{i+4}$, we obtain $\mathcal{S} \cap\left(C_{i+1} \cup \cdots \cup C_{i+4}\right) \mid \geq 7$.

If $s_{3, i+1} \notin \mathcal{S}$, then $s_{2, i+1}$ and $s_{4, i+1}$ belong to $\mathcal{S}$, which contradicts claim (6). Assume now that $s_{3, i+1} \in \mathcal{S}$. This implies that $s_{4, i+2} \in \mathcal{S}$. We may assume that $s_{3, i+2} \notin$ $\mathcal{S}$. Since by Lemma 1 applied on $\left(R_{2} \cup R_{3} \cup R_{4}\right) \cap\left(C_{i+3} \cup C_{i+4}\right)$ and since $\mathcal{S} \cap$ $\left\{s_{2, i+1}, s_{1, i+2}, s_{2, i+2}, s_{1, i+3}, s_{1, i+4}\right\} \neq \emptyset$, we obtain that $\left|\mathcal{S} \cap\left(C_{i+1} \cup \cdots \cup C_{i+4}\right)\right| \geq 7$. We claim that:

We may assume that $\left|\mathcal{S} \cap\left(C_{i+2}-s_{4, i+2}\right)\right|=1,\left|\mathcal{S} \cap C_{i+3}\right|=1$ and $\left.\mid \mathcal{S} \cap C_{i+4}\right) \mid=1$.

If $\mathcal{S} \cap\left(C_{i+2}-s_{4, i+2}\right)=\emptyset$, then $\mathcal{S} \cap C_{i+3} \supseteq\left\{s_{1, i+3}, s_{2, i+3}, s_{3, i+3}\right\}$. Moreover, $\mathcal{S} \cap\left(C_{i+4} \cup\right.$ $\left.\left\{s_{4, i+3}\right\}\right) \neq \emptyset$; and so $\left|\mathcal{S} \cap\left(C_{i+1} \cup \cdots \cup C_{i+4}\right)\right| \geq 7$. If $\left|\mathcal{S} \cap C_{i+2}\right| \geq 3$, then, by Lemma 1 applied on $C_{i+3} \cup C_{i+4}$, we obtain that $\left|\mathcal{S} \cap\left(C_{i+1} \cup \cdots \cup C_{i+4}\right)\right| \geq 7$.

To prove that we may assume $\left|\mathcal{S} \cap C_{i+3}\right|=1$ and $\left.\mid \mathcal{S} \cap C_{i+4}\right) \mid=1$, it is sufficient to observe that $\mathcal{S}$ intersects columns $C_{i+3}, C_{i+4}$ and that $\left|\mathcal{S} \cap\left(C_{i+1} \cup C_{i+2}\right)\right|=4$.

By Lemma 1 applied on $\left(C_{i+3} \cup C_{i+4}\right)-\left\{s, s^{\prime}\right\}$, we can see that we may assume that $s=s_{4, i+3}$ and $s^{\prime}=s_{4, i+4}$ (resp. $s=s_{1, i+3}$ and $\left.s^{\prime}=s_{1, i+4}\right)$ do not belong to $\mathcal{S}$.

This last remark implies that $\mathcal{S}$ intersects both $\left\{s_{2, i+3}, s_{2, i+4}\right\}$ and $\left\{s_{3, i+3}, s_{3, i+4}\right\}$. Combining (7), and the previous remarks, we obtain that the only solutions when $\left|\mathcal{S} \cap\left(C_{i+1} \cup \cdots \cup C_{i+4}\right)\right|<7$ are $\mathcal{S}=\left\{s_{1, i+1}, s_{3, i+1}, s_{2, i+2}, s_{4, i+2}, s_{2, i+3}, s_{3, i+4}\right\}$ and $\mathcal{S}=\left\{s_{1, i+1}, s_{3, i+1}, s_{2, i+2}, s_{4, i+2}, s_{3, i+3}, s_{2, i+4}\right\}$. In any case, $C_{i+4}$ (up to rotation) is respectively a $P(4)$ or a $P(3,4)$ configuration.

Lemma 4. Let $2 \leq i \leq n-4$. If $C_{i-1}$ and $C_{i+4}$ are a $P(4)$ or a $P(3,4)$ configuration of $\mathcal{S}$, then $\left|\mathcal{S} \cap\left(C_{i} \cup C_{i+1} \cup C_{i+2} \cup C_{i+3}\right)\right| \geq 8$.

Proof. First assume that $C_{i}$ and $C_{i+4}$ are $P(3,4)$ configurations.

If $s_{2, i-1} \in C_{i-1}$ and $s_{2, i+4} \in C_{i+4}$, then, similarly as in the proof of Lemma 3, we have that $s_{1, i}, s_{1, i+3}, s_{3, i}, s_{4, i+1}, s_{4, i+2}$ and $s_{3, i+3}$ are in $\mathcal{S}$. We conclude by applying Lemma 1 on $\left(C_{i+1} \cup C_{i+2}\right) \cap\left(R_{1} \cup R_{2} \cup R_{3}\right)$.

If $s_{2, i-1} \in C_{i-1}$ and $s_{3, i+4} \in C_{i+4}$, then, similarly as in the proof of Lemma 3, we have that $s_{1, i}, s_{4, i+3}, s_{3, i}, s_{4, i+1}, s_{1, i+2}$ and $s_{2, i+3}$ are in $\mathcal{S}$. It is now straightforward to check that we need three more squares to exclude all pentominoes in $C_{i-1} \cup C_{i} \cup \cdots \cup C_{i+4}$.

If $C_{i-1}$ is a $P(3,4)$ configuration and $C_{i+4}$ is a $P(4)$ configuration, then, similarly as in the proof of Lemma 3, we have that $s_{1, i}, s_{3, i}$ and $s_{4, i+1}$ are in $\mathcal{S}$. Moreover, since $C_{i+4}$ is a $P(4)$ configuration, $\left|\mathcal{S} \cap C_{i+3}\right| \geq 3$, we conclude by applying Lemma 1 on $\left(C_{i+1} \cup C_{i+2}\right) \cap\left(R_{1} \cup R_{2} \cup R_{3}\right)$.

By symmetry, the lemma holds again if $C_{i+4}$ is a $P(3,4)$ configuration and $C_{i-1}$ is a $P(4)$ configuration.

If $C_{i-1}$ and $C_{i+4}$ are both $P(4)$ configurations, then $\left|\mathcal{S} \cap\left(C_{i} \cup C_{i+3}\right)\right| \geq 6$. Hence, we complete the proof of Lemma 4 by applying Lemma 1 on $\left(C_{i+1} \cup C_{i+2}\right) \cap$ $\left(R_{1} \cup R_{2} \cup R_{3}\right)$. 


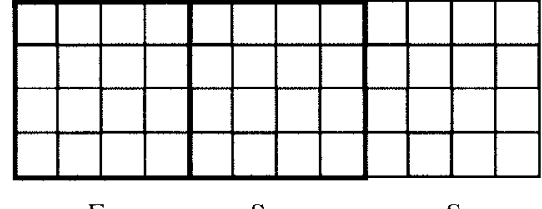

$\mathrm{S}$
$S$

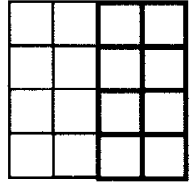

$S$ or

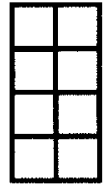

S'

Fig. 8. The solutions of $\left(\mathrm{PEP}_{4 \times n}\right)$.

From Lemmas 3 and 4, we obtain:

Theorem 5. An optimal solution of $\left(\mathrm{PEP}_{4 \times n}\right)$ with $n=4 q \geq 4$ has cardinality $6 q-1$. Moreover, up to rotation, any optimal solution of $\left(\mathrm{PEP}_{4 \times 4 q}\right)$ can be described as shown in Fig. 8.

Proof. We consider an intermediate problem, denoted by $\left(\mathrm{PEP}_{4 \times 4 q}^{\prime}\right)$ : If the column $C_{0}$ is a $P(3,4)$ configuration, then what is the smallest cardinality of a set which excludes all pentominoes in $C_{1} \cup C_{2} \cup \cdots \cup C_{n}$ ?

We claim that:

Any solution $\mathcal{S}$ of $\left(\mathrm{PEP}_{4 \times 4 q}\right)$ has cardinality at least $6 q-1$. Any solution $\mathcal{S}^{\prime}$ of $\left(\mathrm{PEP}_{4 \times 4 q}^{\prime}\right)$ has cardinality at least $6 q$. Moreover, if $|\mathcal{S}|=6 q-1$, then, up to rotation, $C_{4 q}$ is a $P(3,4)$ or a $P(4)$ configuration and $C_{1}$ is a $P(4)$ configuration. If $\left|\mathcal{S}^{\prime}\right|=6 q$, then, up to rotation, $C_{4 q}$ is a $P(4)$ or a $P(3,4)$ configuration.

The proof works by induction on $q$. If $q=1$, then Theorem 5 follows from Lemmas 2 and 3. Assume now that $q>1$.

Case A: $\mathcal{S}$ is a solution to $\left(\mathrm{PEP}_{4 \times 4 q}\right)$. Let $A_{1}, \ldots, A_{q}$ be a partition of $G_{4, n}$ where the $A_{i}$ 's are blocks of four consecutive columns. Observe that, by the induction hypothesis, we have $\left|\mathcal{S} \cap\left(A_{2} \cup \cdots \cup A_{q}\right)\right| \geq 6(q-1)-1,\left|\mathcal{S} \cap A_{1}\right| \geq 5$.

Subcase A.1: $\left|\mathcal{S} \cap\left(A_{2} \cup \cdots \cup A_{q}\right)\right|>6(q-1)-1$. If $\left|\mathcal{S} \cap\left(A_{2} \cup \cdots \cup A_{q}\right)\right|>$ $6(q-1)$, then, by Lemma 2 , we obtain $|\mathcal{S}| \geq 6 q$, and so the claim holds. Assume that $\left|\mathcal{S} \cap\left(A_{2} \cup \cdots \cup A_{q}\right)\right|=6(q-1)$. If $\left|\mathcal{S} \cap A_{1}\right|>5$, then the claim holds similarly as in the previous case. So, we may assume that $\left|\mathcal{S} \cap A_{1}\right|=5$. Hence, $|\mathcal{S}|=6 q-1$.

If $\left|\mathcal{S} \cap A_{2}\right| \geq 7$, then, by the induction hypothesis applied on columns $A_{3}, \ldots, A_{q}$ and since $|\mathcal{S}|=6 q-1$, we obtain $\left|\mathcal{S} \cap A_{2}\right|=7$ and $\left|\mathcal{S} \cap\left(A_{3} \cup \cdots \cup A_{q}\right)\right|=6(q-2)-1$. However, now by the induction hypothesis applied on columns $A_{3}, \ldots, A_{q}$, we have that $\mathcal{S} \cap A_{3}$ is either a $P(4)$ or a $P(3,4)$ configuration. So, in any case, this contradicts Lemma 4.

Thus, by Lemma 3, we have $\left|\mathcal{S} \cap A_{2}\right|=6$ and so $\mathcal{S} \cap C_{4}$ is a $P(3,4)$ configuration. Observe that $\mathcal{S} \cap\left(A_{2} \cup \cdots \cup A_{q}\right)$ is a solution $\mathcal{S}^{\prime}$ of $\left(\mathrm{PEP}_{4 \times 4 q}^{\prime}\right)$ of cardinality $6(q-1)$. Thus, by the induction hypothesis, claim (8) holds.

Subcase A.2: $\left|\mathcal{S} \cap\left(A_{2} \cup \cdots \cup A_{q}\right)\right|=6(q-1)-1$. Without loss of generality, we may assume that $\left|\mathcal{S} \cap A_{1}\right|=6$. Moreover, by the induction hypothesis, we have either $\left|\mathcal{S} \cap A_{2}\right|=5$ or $\left|\mathcal{S} \cap A_{q}\right|=5$. 
If $\left|\mathcal{S} \cap A_{2}\right|=5$, then we may assume $q>2$ for otherwise we conclude as in Subcase A.1. Hence, $\mathcal{S} \cap A_{2}$ is a $P(4)$ configuration. Thus, $\left|\mathcal{S} \cap A_{1}\right|=6$ contradicts Lemma 3.

If $\left|\mathcal{S} \cap A_{2}\right|=6$, then, by Lemma $3, \mathcal{S} \cap A_{2}$ is a $P(3,4)$ configuration. So claim (8) holds by Lemma 3.

Case B: $\mathcal{S}$ is a solution to $\left(\mathrm{PEP}_{4 \times 4 q}^{\prime}\right)$. Let $A_{1}, \ldots, A_{q}$ be a partition of $G_{4, n}$ where the $A_{i}$ 's are blocks of four consecutive columns. Observe that by the induction hypothesis we have $\left|\mathcal{S} \cap\left(A_{2} \cup \cdots \cup A_{q}\right)\right| \geq 6(q-1)-1,\left|\mathcal{S} \cap A_{1}\right| \geq 6$.

If $\left|\mathcal{S} \cap A_{2} \cap \cdots \cap A_{q}\right|>6(q-1)-1$ and if $\left|\mathcal{S} \cap A_{1}\right|>6$, then clearly the claim holds. If $\left|\mathcal{S} \cap A_{1}\right|=6$, then, by Lemma $3, \mathcal{S} \cap\left(A_{2} \cup \cdots \cup A_{q}\right)$ is a solution of $\left(\operatorname{PEP}_{4 \times 4(q-1)}^{\prime}\right)$. So claim (8) holds, by the induction hypothesis.

Suppose that $\left|\mathcal{S} \cap\left(A_{2} \cup \cdots \cup A_{q}\right)\right|=6(q-1)-1$. By the induction hypothesis, we have either $\left|\mathcal{S} \cap A_{2}\right|=5$ or $\left|\mathcal{S} \cap A_{q}\right|=5$.

If $\left|\mathcal{S} \cap A_{2}\right|=5$, then $C_{5}$ is a $P(4)$ configuration and so, by Lemma $4,\left|\mathcal{S} \cap A_{1}\right| \geq 8$. Hence, claim (8) holds.

If $\left|\mathcal{S} \cap A_{q}\right|=5$, then $C_{5}$ is a $P(4)$ or a $P(3,4)$ configuration and so, by Lemma 4 and since $C_{0}$ is a $P(3,4)$ configuration, we obtain again that $\left|\mathcal{S} \cap A_{1}\right| \geq 8$, which completes the proof of claim (8).

Now, as in the proof of claim (8), we have that any optimal solution $\mathcal{S}$ of $\left(\mathrm{PEP}_{4 \times 4 q}\right)$ has cardinatity $6 q-1$ and is obtained as shown in Fig. 8.

Using the same technique employed in the proofs of Lemmas 2-4, we can prove that any solution $\mathcal{S}$ and $\mathcal{S}^{\prime}$ of $\left(\mathrm{PEP}_{4+r}\right)$ and respectively $\left(\mathrm{PEP}_{4+r}^{\prime}\right)$ satisfies

$$
\left|\mathcal{S} \cap\left(C_{1} \cup \cdots \cup C_{4+r}\right)\right| \geq\left\{\begin{array}{lll}
7 & \text { if } & r=1, \\
8 & \text { if } & r=2, \\
10 & \text { if } & r=3,
\end{array}\right.
$$

and $\left|\mathcal{S}^{\prime} \cap\left(C_{1} \cup \cdots \cup C_{4+r}\right)\right| \geq\left|\mathcal{S} \cap\left(C_{1} \cup \cdots \cup C_{4+r}\right)\right|+1$.

Finally, by a simple induction and using Theorem 5 , we can prove that

$$
\mathcal{S} \cap G_{4,4 q+r}=\left\{\begin{array}{lll}
6 q-1 & \text { if } & r=0 \\
6 q+1 & \text { if } & r=1 \\
6 q+2 & \text { if } & r=2 \\
6 q+4 & \text { if } & r=3
\end{array}\right.
$$

Unfortunately, for $r \neq 0$, the optimal solutions are not "unique."

\section{Concluding Remarks}

It is straightforward from Lemmas 2 and 3 to prove that any solution of $\left(\mathrm{PEP}_{8 \times 8}\right)$ has at least 24 squares. We do not give the details of the proof here since Bosch [1] solved it using a computer and an integer linear programming approach. Our approach should be helpful to solve ( $\left.\mathrm{PEP}_{k \times n}\right)$-type problems for any fixed $k$. Another family of problems should be to consider other lattices instead of the chessboard (grid). 


\section{References}

1. R. A. Bosch, A Pentomino Exclusion Problem. Mathematical Programming Newsletter, Optima 60 (December 1998), 3.

2. S. W. Golomb, Polyominoes - Puzzles, Patterns, Problems, and Packings. Princeton Science Library, Princeton, NJ (1994).

3. S. Gravier and C. Payan, Tilings and Isoperimetrical Shapes. Submitted (1999).

4. H. Melissen, Packing and Covering with Circles. Ph.D. Thesis, Proefschrift Universiteit Utrecht (1997).

Received June 7, 1999, and in revised form March 1, 2001. Online publication August 9, 2001. 